\title{
Market clearing model of distribution network-side integrated energy system
}

\author{
Dongmei Yang ${ }^{1}$, Yong $\mathrm{Sun}^{2}$, Baoju $\mathrm{Li}^{2}$, Xiaochen Zhang ${ }^{1}$, Pengfei $\mathrm{Li}^{3,{ }^{*}}$ and Dunnan $\mathrm{Liu}^{3}$ \\ ${ }^{1}$ NARI Group Corporation (State Grid Electric Power Research Institute), Nanjing 211106, Jiangsu Province, China \\ ${ }^{2}$ State Grid Jilin Electric Power Company Limited, Changchun 130021, Jilin Province, China \\ ${ }^{3}$ North China Electricity Power University, Beijing, 102206, China
}

\begin{abstract}
In an electric-gas integrated energy system that considers the coupling conditions between systems, there is energy transaction between the systems. So the node energy price setting method and market clearing strategy of the coupled system are worth studying. First of all, this paper establishes a market clearing model for electricity / gas subsystems and a method for solving node energy prices. Then, a market clearing model of the electrical coupling system is established based on this. Finally, an example to verify the effectiveness of the nodal energy price formulation method and the market clearing model is given.
\end{abstract}

\section{Introduction}

The electric-gas-heat integrated energy system can supply power, gas and heat at the same time. And with its high efficiency, low emissions, and various operating modes, it has attracted widespread attention both at home and abroad. In the future energy trading market, the optimization of production methods and pricing strategies of integrated energy systems is the hot spot for manufacturers and users. Besides, in the energy market, users with multiple energy needs will adjust and determine their demands according to the price of different energy sources in order to maximize their own benefits. And in energy interactions, the behaviors and strategies of energy producers and users will affect the interests and strategies of other participants, which will enable interaction and competition among market participants and improve the efficiency of resource allocation in the energy market.

In the market clearing problem of electricity / gas / heating subsystem, since there is no coupling constraint between the systems, each system only needs to consider the problem of interaction equilibrium between the energy supplier and the user1. Then according to the optimized operating conditions of each system, the node prices at market clearing can be solved; in the electricgas-heat integrated energy system that considers the coupling conditions between the systems, there is an energy transaction between the systems because of the existence of energy conversion equipment. This moment, the node energy price setting method and the market clearing strategy of the coupled system are worth studying. Therefore, this paper studies the market clearing model and the node energy price setting method under the coupling of electrical system.

\section{Market clearing model of electricity I gas subsystems}

This section mainly introduces how to set up a market clearing model for electricity / gas subsystems and how to solve the nodal energy prices. References [3-5] list the objective functions and various constraints for the optimal operation of each system.

\subsection{Market clearing model of power system}

In the power system optimization operation model, rational power producers aim to reduce costs. So the objective function is to minimize the total running cost, and the constraint conditions include the node power balance equation, the generator output upper and lower limit constraints, the generator output climbing power constraint, the line transmission active power and the relationship between the phase angle at both ends of the line, and the line transmission power constraint, etc. It can be specifically described as follows:

$$
\begin{gathered}
\min \sum_{t \in \mathcal{T}}\left(\sum_{g \in \mathcal{G}_{n}} p_{g t} C_{g}\right) \\
\text { s.t. } \sum_{g \in \mathcal{G}_{n}} p_{g t}+\sum_{r \in \mathcal{R}_{n}} p_{r t}-\sum_{l \in \mathcal{L}_{n}^{+}} p_{l t}+\sum_{l \in \mathcal{L}_{n}} p_{l t}-\sum_{d \in \mathcal{D}_{n}} p_{d t}=0: \lambda_{p}, \forall n, t \\
P_{g}^{\min } \leq p_{g t} \leq P_{g}^{\max }, \forall g, t \\
-R_{g}^{D} \leq p_{g t}-p_{g(t-1)} \leq R_{g}^{U}, \forall g, t
\end{gathered}
$$

\footnotetext{
Corresponding author: 2210055766@qq.com
} 


$$
\begin{gathered}
-P_{l}^{\max } \leq p_{l t} \leq P_{l}^{\max }, \quad \forall l, t \\
p_{l t}=\frac{\theta_{l^{+} t}-\theta_{l^{-} t}}{X_{l}}, \quad \forall l, t
\end{gathered}
$$

In the above expressions, equation (1) is the objective function that is minimizing the total operating cost of the power system, and equations (2)-(6) are the operating constraints of the power system. In formula (1), $\mathrm{p}_{\mathrm{gt}}$ represents the output of the generator, and $\mathrm{C}_{\mathrm{g}}$ represents the power generation cost of the unit. Formula

(2) represents the node power balance equation. $p_{\text {rt }}$ represents the output of the distributed generation device,

$p_{l t}$ represents the power flowing on the line, and $p_{d t}$ represents electric load power. Formula (3) represents the upper and lower limits of the generator output. ${ }_{\mathrm{g}}{ }^{\max }$ and $\mathrm{P}_{\mathrm{g}}^{\mathrm{min}}$ repr of the generator output. Formula (4) represents the power limitation of the generator to climb the hill. $\mathrm{R}_{\mathrm{g}}^{\mathrm{U}}$ and $\mathrm{R}_{\mathrm{g}}^{\mathrm{D}}$ represent the limits of the climbing power up and down respectively. Formula (5) represents the active power constraint of the line transmission. $\mathrm{P}_{1}^{\max }$ represents the maximum current carrying capacity of the line. Formula (6) represents the constraint on the relationship between the transmission active power of the line and the phase angle at both ends of the line. $\mathrm{X}_{\mathrm{l}}$ represents the line reactance. Besides, $\theta_{1^{+}} \mathrm{t}$ and $\theta_{1^{-}} \mathrm{t}$ represent the voltage phase angles of the first and last nodes of the line respectively. In addition, the dual variables of the corresponding constraints are listed to the right of each constraint.

The node electricity price is a Lagrangian multiplier (that is, dual variable) of the node power balance equation. Therefore, it is necessary to set up the dual problem of the optimal operation of the power system and solve the dual variable.

\subsection{Natural Gas System Market Clearance Model}

In the optimized operation model of the natural gas system, rational gas producers aim to reduce costs. Therefore, the objective function is to minimize the total operating cost, and the constraints include the nodal flow balance equation, the natural gas supply flow restriction, nodal pressure value constraint, natural gas energy storage constraint, compressor constraint, and the constraints between pipeline flow and pressure at both ends. It can be described as follows:

$$
\begin{gathered}
\min \sum_{t \in \mathcal{T}}\left(\sum_{s \in \mathcal{S}_{n g}} f_{s t} C_{s}\right) \\
\text { s.t. } \sum_{s \in \mathcal{S}_{n g}} f_{s t}-\sum_{p \in \mathcal{P}_{n g}^{+}} f_{p^{+} t}+\sum_{p \in \mathcal{P}_{n g}^{-}} f_{p^{-} t}-\sum_{d_{g} \in \mathcal{D}_{\mathcal{G}}\left(d_{g}\right)} f_{d_{g} t}=0, \quad \forall n_{g}, t
\end{gathered}
$$

$$
\begin{gathered}
F_{s}^{\min } \leq f_{s t} \leq F_{s}^{\max }, \forall s, t \\
\tau_{n_{g}}^{\min } \leq \tau_{n_{g} t} \leq \tau_{n_{g}}^{\max }, \quad \forall n_{g}, t \\
\tau_{c^{+} t} \leq \tau_{c^{-}, t} \leq Z_{c} \tau_{c^{+} t^{+}}, \forall c, t \\
f_{c t} \geq 0, \quad \forall c, t \\
f_{p t}\left|f_{p t}\right|=\varphi\left(\tau_{p^{+} t}^{2}-\tau_{p^{-} t}^{2}\right), \forall p, t
\end{gathered}
$$

In the above expressions, formula (7) is the objective function, which is to minimize the total operating cost of the natural gas system, and Formula (8)-(13) are the operating constraints of the natural gas system. In formula (7), $f_{\text {st represents the outflow of the natural gas }}$ source node, and $\mathrm{C}_{\mathrm{s}}$ represents the gas production cost of the gas well. Formula (8) represents the node flow balance equation. $\mathrm{f}_{\mathrm{p}^{+} \mathrm{t}}$ and $f_{p-t}$ represent the natural gas flow at the head and end of the pipeline, and $f_{d_{g}} t$ represents the natural gas load flow. Formula (9) represents the natural gas flow restriction. $\mathrm{F}_{\mathrm{s}}^{\mathrm{min}}$ and $\mathrm{F}_{\mathrm{s}}^{\max }$ represent the lower flow limit and upper flow limit of the natural gas source respectively. Formula (10) represents the node pressure constraint. $\tau_{n_{\mathrm{g}}}^{\min }$ and $\tau_{\mathrm{n}_{\mathrm{g}}}^{\max }$ represent the minimum and maximum values of the node natural gas pressure respectively. Formula (11) represents the compressor constraint. $\boldsymbol{\tau}_{\mathrm{c}}{ }^{+} \mathrm{t}$ and $\boldsymbol{\tau}_{\mathrm{c}^{-} \mathrm{t}}$ respectively represent the natural gas pressure at the head and end of the pipeline where the compressor is located, and $\mathrm{Z}_{\mathrm{c}}$ represents the maximum compression ratio of the compressor. Formula (12) indicates that the flow of the pipeline where the compressor is located is non-negative. $\mathrm{f}_{\mathrm{ct}}$ indicates the flow through the compressor. Formula (13) represents the relationship constraint between the pipeline flow and the pressure at both ends. $\emptyset$ represents the relationship coefficient between the pipeline flow and the squared pressure difference between the first and last nodes.

The node gas price and the node electricity price are solved in the same principle, and both require the establishment of a dual problem to solve the Lagrange multiplier of the node flow balance equation.

\section{Market clearing model of electric- pneumatic coupling system}

In an integrated energy system, various energy sources can be converted to each other. For example, gas turbines can convert natural gas into electricity, and some electricity-to-gas equipment can use excess electricity to produce natural gas and hydrogen. 


\subsection{Electricity market clearing model in electric- pneumatic coupling system}

This section mainly studies the power market clearing model in the electric-pneumatic coupling system. In the electric-pneumatic coupling system, the power system and the natural gas system are coupled by a gas turbine. From the perspective of the power market clearing, compared with the independent power market clearing, the coupled clearing increases the gas purchase cost in terms of cost, and the total cost is shown in equation (14). In terms of constraints, gas turbine operating limits have been increased, such as constraints on the upper and lower limits of power generation and constraints on climbing. As shown in equations (15)-(16). So the power market clearing model when coupled can be expressed by equations -and (14)-(16).

$$
\begin{gathered}
\min \left[\sum_{g \in \mathcal{G}_{n}} p_{g t} C_{g}+\sum_{n \in \mathcal{N}}\left(\sum_{m \in \mathcal{M}} f_{m t} \lambda_{n}\right)\right] \\
P_{m}^{\min } \leq p_{m t} \leq P_{m}^{\max }, \forall m, t \\
-\boldsymbol{R}_{m}^{D} \leq p_{m t}-p_{m(t-1)} \leq \boldsymbol{R}_{m}^{U}, \quad \forall m, t
\end{gathered}
$$

In formula (14), $\mathrm{f}_{\mathrm{mt}}$ is the flow of natural gas consumed by the gas turbine, and ${ }^{\lambda} \mathbf{n}$ is the serial number of the natural gas node connected to the gas turbine. In formula (15), $\mathrm{p}_{\mathrm{mt}}$ indicates the power generation of the gas turbine, and $\mathrm{P}_{\mathrm{m}}^{\min }$ and $\mathrm{P}_{\mathrm{m}}^{\max }$ respectively indicate the upper and lower limits of the power generation of the gas turbine. In formula (16), $\mathrm{R}_{\mathrm{m}}^{\mathrm{D}}$ and $\mathrm{R}_{\mathrm{m}}^{\mathrm{U}}$ respectively represent the downhill climbing power and the uphill climbing power limit of the gas turbine.

\subsection{Natural gas market clearing model in electric-pneumatic coupling system}

This section mainly studies the natural gas market clearing model in the electric-pneumatic coupling system. The specific relationship is shown in Equation (17).

$$
f_{m t}=P_{m t} /\left(K_{g} \eta_{m}\right)
$$

Among them, $\mathrm{P}_{\mathrm{mt}}$ indicates the power generation of the gas turbine, $\mathrm{K}_{\mathrm{g}}$ indicates the calorific value of natural gas, and ${ }^{\eta} \mathrm{m}$ indicates the efficiency of the gas turbine. Therefore, the coupled natural gas market clearing model can be expressed by equations (7)-(13) and (17).

\section{The Example Analysis}

In this section, an IEEE-5 node power system and a 7node natural gas system will be constructed, and then coupled through a gas turbine. The coupling system topology is shown in Figure 1, and Table 1 shows the parameters of the gas turbine.

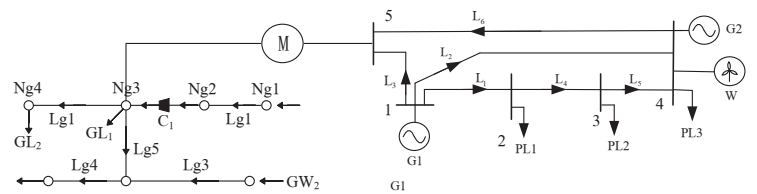

Fig. 1. Topology diagram of electric-pneumatic coupling system

Table 1. Parameters of gas turbine in electric-pneumatic coupling system

\begin{tabular}{|c|c|}
\hline Parameter & $\begin{array}{c}\text { Gas } \\
\text { Turbine }\end{array}$ \\
\hline $\begin{array}{c}\text { Ordinal Number of Nodes connected to the } \\
\text { Power System }\end{array}$ & 5 \\
\hline $\begin{array}{c}\text { Ordinal Number of Nodes connected to the } \\
\text { Natural Gas System }\end{array}$ & 3 \\
\hline Maximum Output & $400 \mathrm{MW}$ \\
\hline Minimum Output & $180 \mathrm{MW}$ \\
\hline Climbing Power Up & $100 \mathrm{MW} / \mathrm{h}$ \\
\hline Climbing Power Down & $100 \mathrm{MW} / \mathrm{h}$ \\
\hline Effectiveness & 0.4 \\
\hline
\end{tabular}

\subsection{Electricity market clearing results in the electric-pneumatic coupling system}

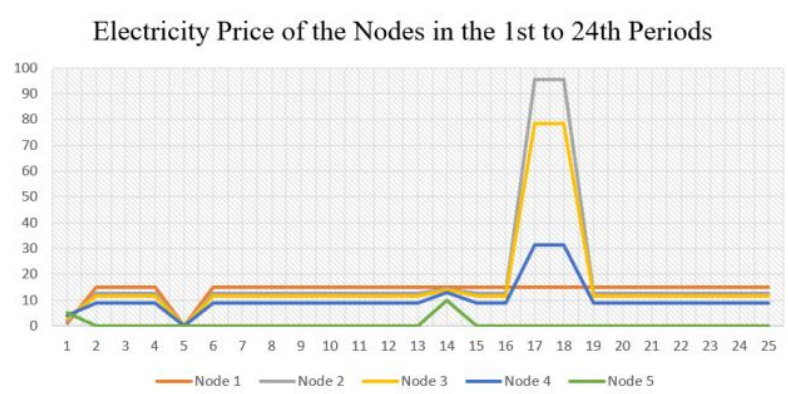

Fig. 2. Electricity Price of the Nodes in the 1st to 24th Periods 


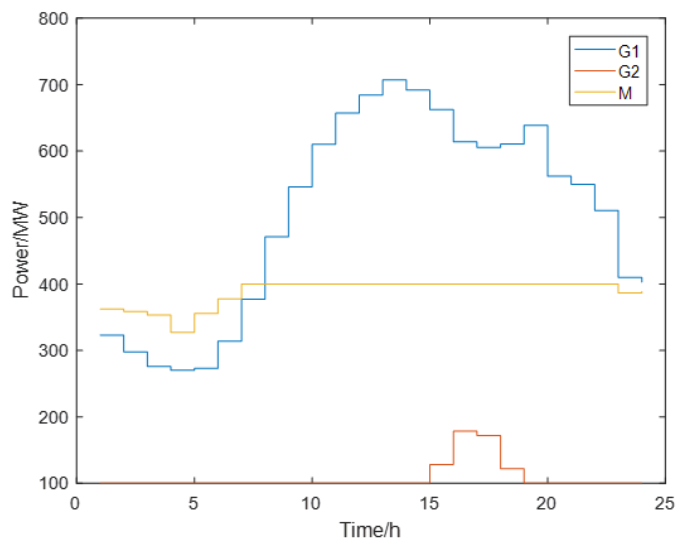

Fig. 3. Graph of generator output in electric-pneumatic coupling system

It can be concluded through analysis that the electricity price of the node has changed at first because of the introduction of gas turbines, and the decrease in the numerical value of the optimization result (that is, the overall cost) can reflect the reduction of the total cost. Besides, the gas turbine's power output is close to that of the G3 generator, and the overall operating state is in line with the real-time balance of supply and demand in the power system. It can be seen from the parameter table that the technical parameters of the gas turbine are close to G3. However, because the cost of natural gas consumption is relatively low, the total cost is reduced, which also reduces the electricity price of some nodes to the cost of gas purchase, or take the gas purchase cost as the lower price limit.

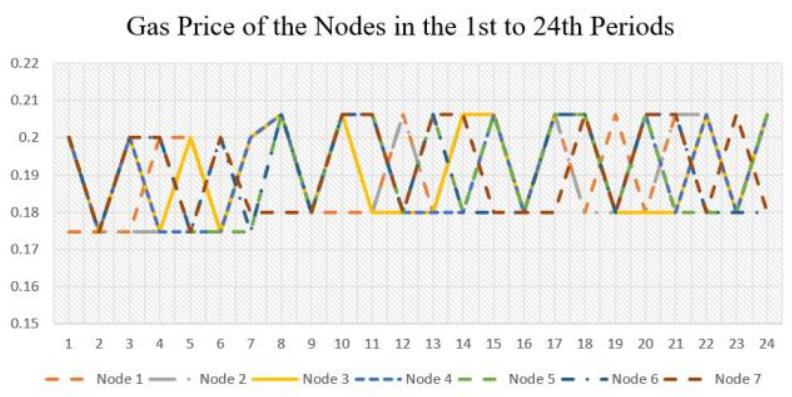

Fig. 4. Gas Price of the Nodes in the 1st to 24th Periods

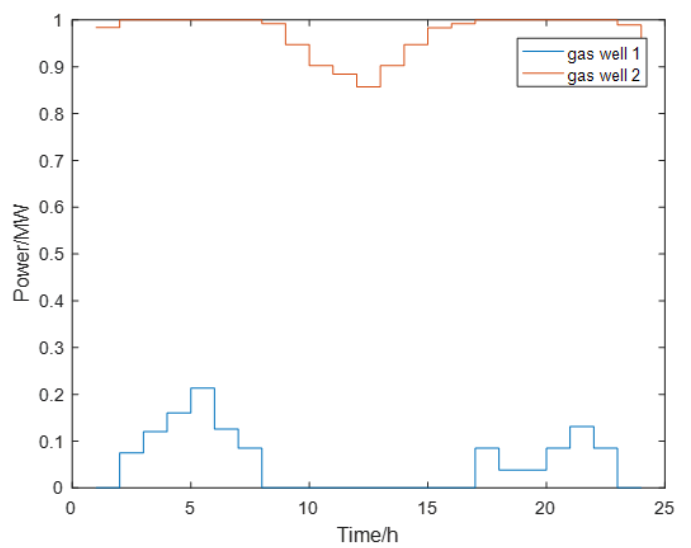

Fig. 5. Flow curve of gas produced by gas well in electricpneumatic coupling system
In this example, the gas turbine is connected to the gas network node 3, which leads to an increase in the total cost of the natural gas system at first. That's because the total gas load is increased after the gas turbine is connected, and the total gas production from the gas well is changed too. And in the stand-alone natural gas system, the fluctuation of the original gas load at node 3 is different from the fluctuation trend of the gas consumption of the gas turbine. Because the output fluctuation of the gas turbine in the power system determines the fluctuation trend of the gas consumption of the gas turbine, which changes the trend of gas production from gas wells. Therefore, it is different from simply increasing the total air load of this node.

\section{Conclusion}

In this paper, a market clearing model of the electric / gas subsystem is established, and a method for solving the node energy price of the electric-pneumatic coupling system is proposed, which enables each system to have the ability to conduct energy transactions with other systems when there are conditions for coupling. Then through the analysis of specific examples to verify the model and propose a market clearing strategy for integrated energy systems. By comparing the solution results of the gas turbine under different operating conditions, it can be seen that the operating status of the coupling element has an impact on market behavior and clearance results.

\section{Acknowledgements}

Science and Technology Project of SGCC, Research and Demonstration of Integrated Energy Management and Optimal Control Technology for Multi-energy Flow.

\section{References}

1. H.B. Sun, Z.G Pan, Q.L. Guo. Automation of Electric Power Systems, Research on Multi-energy Flow Energy Management: Challenges and Prospects, 15, 1(2016)

2. J. Wang, W. Gu, S. Lu, et al. Automation of Electric Power Systems, Collaborative planning of multiregion integrated energy system combined with heat network model, 15,17(2016)

3. Z.N. Wei, S.D. Zhang, G.Q Sun, et al. Chinese Journal of Electrical Engineering, Research on Peak-cutting and Valley-filling of Electricity-gas Interconnected Integrated Energy System Considering Electricity to Gas, 16, 4601(2017)

4. L.R. Deng, H.B. Sun, R.Z. Chen, et al. Power System Technology, Research on Energy Price of Cogeneration System for Energy Internet, 11, 3375(2016).

5. X.D. Yuan, Z.G. Yu, Y.Y Zhang, et al. Global Energy Internet, Distribution network optimization taking into account photovoltaic interval prediction and energy storage SOC equilibrium, 2,598(2019). 